\title{
Abdominal Aortic Aneurysm in Pre-term Pregnancy: A Case Report
}

\author{
Rafa Jireh O. Iglesias, MD, MBA and Maria Teresita B. Aspi, MD, DPBA \\ Department of Anesthesiology, Philippine General Hospital, University of the Philippines Manila
}

\begin{abstract}
Cardiovascular diseases during pregnancy account for significant morbidity and mortality. An abdominal aortic aneurysm posts high mortality for otherwise healthy patients, more so for conditions that alter normal physiology such as in preterm pregnancy. Abdominal aortic dissection during pregnancy is a rare and life-threatening condition for both the mother and the fetus. An understanding of physiologic maternal changes and surgical stress responses is important to attenuate perioperative hemodynamic changes and prevent progression of aortic expansion and aortic rupture. As an anesthesiologist, one is positioned to facilitate communication among the internist, obstetrician and vascular surgeon for surgical success. This case report presents the anesthetic considerations in the perioperative management of a preterm pregnancy for a major abdominal surgery.
\end{abstract}

Key Words: parturient, abdominal aortic aneurysm, obstetric anesthesia

\section{INTRODUCTION}

Cardiovascular diseases account for significant morbidity and mortality that complicates pregnancy. Abdominal aortic aneurysm belongs in the top list of high mortality vascular cases. ${ }^{1}$ Abdominal aortic dissection in pregnancy is a rare and life-threatening combination for both the mother and fetus. It typically occurs in the third trimester of pregnancy or during the early postpartum period. Back pain presents in $55 \%$ of cases while chest pain is seen in $12 \%$ of patients. ${ }^{2}$ The incidence of rupture is estimated to be at 5.5 per million during pregnancy and post-partum. ${ }^{3}$ The overall mortality rate in an elective uncomplicated operative patient, i.e., absence of rupture or dissection, is less than $7 \%$ while complicated cases approaches $90 \% .{ }^{4}$ Risk factors for worsening of aortic aneurysms include age greater than 75 years, family history, hypertension and Caucasian descent. ${ }^{5}$

\section{CASE PRESENTATION}

This is a case of an otherwise healthy 27-year-old G2P1(1001) parturient with a diagnosis of pregnancy uterine, 27 and 5/7 weeks age of gestation by amenorrhea,

Presented as interesting case at the Society for Obstetric Anesthesia of the Philippines (SOAP) at B. Braun Bonifacio Global Center, Philippines last November 18, 2017.

Corresponding author: Rafa Jireh O. Iglesias, MD, MBA Department of Anesthesiology Philippine General Hospital University of the Philippines Manila

Taft Avenue, Manila 1000, Philippines

Email: rjoiglesias@yahoo.com cephalic presentation, and not in labor. She presented with a one-month history of intermittent flank discomfort. This was initially managed with antibiotics as a case of urinary tract infection for 7 days but without relief of symptoms. On follow-up, abdominal ultrasound was done which showed an incidental finding of a saccular outpouching along the anterior abdominal aortic wall measuring $6.3 \times 6.4 \times$ $7.2 \mathrm{~cm}^{3}$ exhibiting turbulent flow within (Figure 1). 


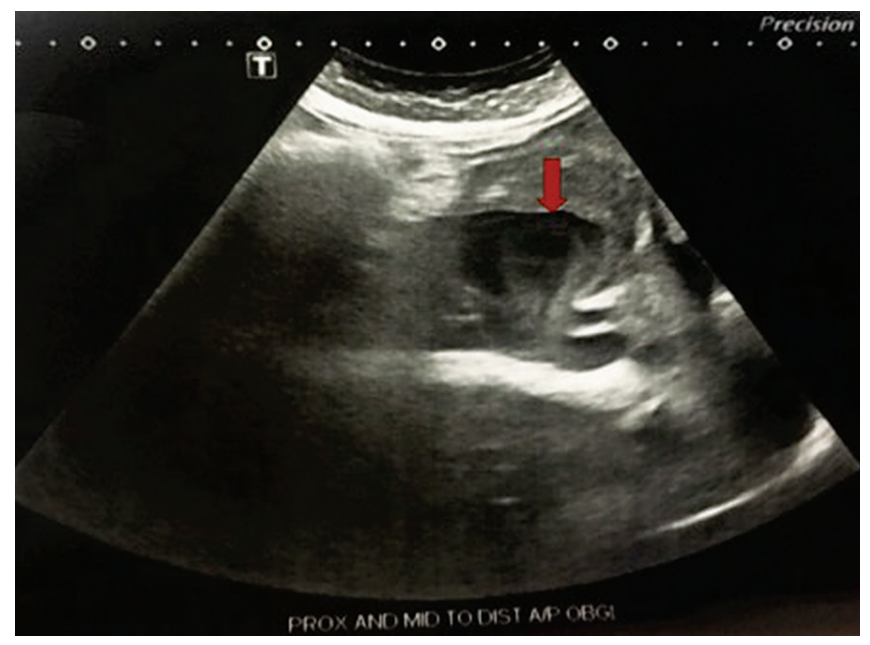

Figure 1. Saccular outpouching along the anterior abdominal aortic wall at the level of the mid to inferior aorta measuring $6.3 \times 6.4 \times 7.2 \mathrm{~cm}$ with maximum thickness of $2.1 \mathrm{~cm}$ exhibiting turbulent flow.

The case was referred to vascular surgery. Because of the size of the aneurysm, it was determined that repair of the aneurysm be scheduled with the surgical delivery of the fetus. The patient was admitted to an intensive maternal unit to optimize the condition of the mother and the baby until term with serial monitoring of the abdominal mass. On $6^{\text {th }}$ day of admission, the patient complained of abdominal pain that prompted a repeat ultrasound. An enlarging pulsatile mass $\left(7.7 \times 7.8 \times 5.9 \mathrm{~cm}^{3}\right)$ and a large hematoma $3 \mathrm{~cm}$ inferior to the renal artery were noted. The aneurysm started to dissect. The patient was scheduled for emergency cesarean section and repair of abdominal aortic aneurysm.

Anesthesia was done with the goal of maintaining stable hemodynamics despite the changes that come with pregnancy, patient positioning, anesthetic technique, and stress of surgery. The planned anesthesia technique was combined epidural anesthesia and general anesthesia.

The patient was received in the operating room with blood pressure of $180 / 70 \mathrm{mmHg}$, heart rate of 80 per minute, respiratory rate of 20 per minute, and oxygen saturation of 99\%. Sudden increase or decrease in hemodynamic status brought by sympathetic activation from anxiety or pain may aggravate the dissecting aneurysm. The patient was sedated with Midazolam $1 \mathrm{mg}(0.02 \mathrm{mg} / \mathrm{kg})$ and Fentanyl $12.5 \mathrm{mcg}$ $(0.21 \mathrm{mcg} / \mathrm{kg})$ prior to the placement of an arterial line for blood pressure monitoring and internal jugular catheter for central venous pressure monitoring. A forced-air warming device was placed to keep the patient thermoregulated. Nitroglycerin via infusion pump was started at $0.5 \mathrm{mcg} /$ $\mathrm{kg} / \mathrm{min}$ for blood pressure control. She was positioned carefully in a left lateral decubitus and an epidural catheter was inserted at the T12 and L1 interspace. Lidocaine 2\% was loaded after negative test dose to the desired block height of T6.
To minimize fetal exposure to anesthesia, surgical preparation with antiseptic solution and placement of drapes were done prior to induction of general anesthesia. The patient was pre-oxygenated with $100 \%$ oxygen and induced with additional Midazolam $2 \mathrm{mg}(0.03 \mathrm{mg} / \mathrm{kg})$, Fentanyl $50 \mathrm{mcg}(0.83 \mathrm{mcg} / \mathrm{kg})$, and Rocuronium $60 \mathrm{mg}(1 \mathrm{mg} / \mathrm{kg})$. Intubation was done and cesarean section immediately commenced. The total time from cutting to delivery of the newborn and placenta was three minutes.

The delivered baby boy was $1,100 \mathrm{~g}$, appropriate for gestational age, and had an APGAR score of 7 and 8 on first and fifth minutes, respectively. The newborn was transferred to the Neonatal Intensive Care Unit for observation. Immediately after delivery, Sevoflurane was turned on and titrated; however, this resulted in hypotension. Nitroglycerin was then stopped to maintain mean arterial pressure of $70-90 \mathrm{mmHg}$. The total time for cesarean section was one hour with an estimated blood loss of $500 \mathrm{ml}$. Packed red blood cells (PRBC) were transfused to replace the blood loss and increase the blood volume in preparation for the aortic aneurysm repair (Figure 2). In anticipation of the expected hypovolemia, Dopamine $(5 \mathrm{mcg} / \mathrm{kg} / \mathrm{min})$ was started to increase the blood pressure at $100 \mathrm{mmHg}$ while preserving renal perfusion.

At the start of aneurysm repair, the systolic blood pressure was down to $70 \mathrm{mmHg}$ from the synergistic effect of sympathetic blockade from epidural anesthesia, and general anesthesia; thus, epidural anesthesia was discontinued. Sevoflurane was turned off and the technique was converted to total intravenous anesthesia with Fentanyl

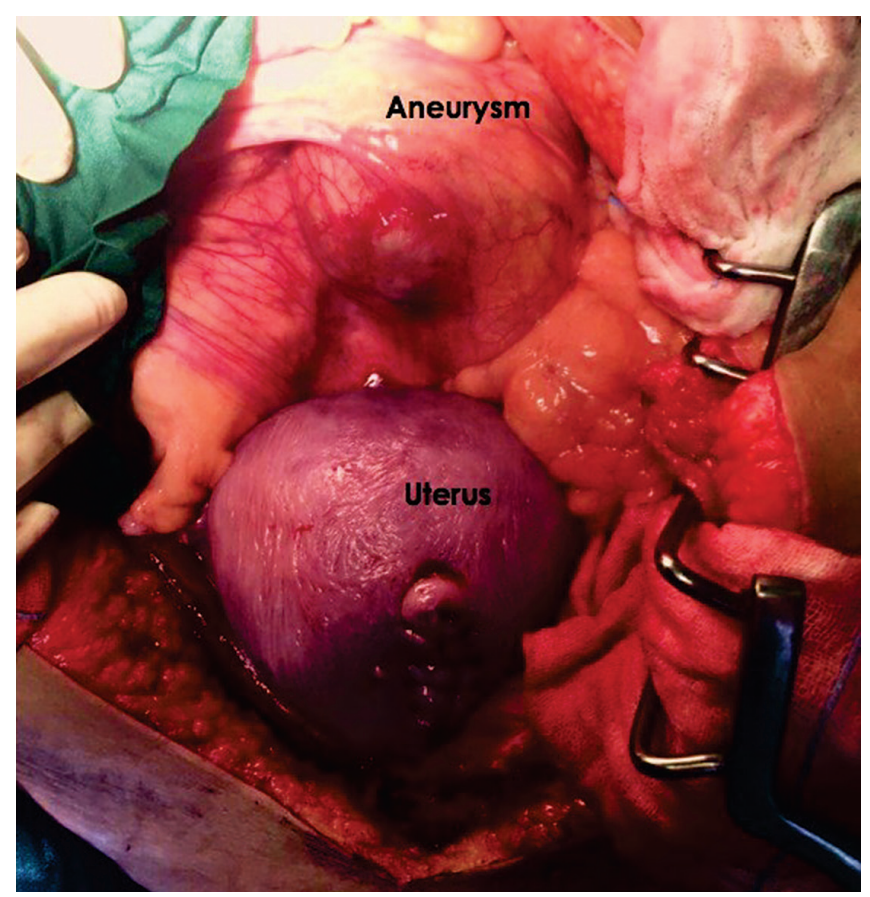

Figure 2. Shows the aneurysm above the uterus after delivery of the baby and closure. 
$(0.5 \mathrm{mcg} / \mathrm{kg})$ every 30 minutes, and Midazolam $(0.04 \mathrm{mg} / \mathrm{kg})$ and Rocuronium $(0.2 \mathrm{mg} / \mathrm{kg})$ every hour. Norepinephrine $(0.05-5 \mathrm{mcg} / \mathrm{kg} / \mathrm{min})$ was started and titrated to augment the blood pressure. During cross-clamping of the abdominal aorta, there is an expected increase in blood pressure from the consequent increase in afterload. In this case, the patient had persistent hypotension; hence, instead of the usual nitroglycerin infusion to help decrease the blood pressure during cross clamping, dopamine and norepinephrine were maintained and titrated. The estimated blood loss was 3,000 $\mathrm{mL}$ and profound hypovolemia was entertained.

Blood loss was replaced with both blood products and crystalloids. Three units of whole blood, 7 units of PRBC, 3 units of fresh frozen plasma and 7 units of platelet concentrate were transfused. Both norepinephrine and dopamine were stopped with recovery of blood pressure at $120 / 60 \mathrm{mmHg}$ and inhalational general anesthesia with Sevoflurane was restarted. Intravenous doses of Midazolam, Fentanyl and Rocuronium were regularly given until the end of the surgery. Paracetamol $900 \mathrm{mg}(18 \mathrm{mg} / \mathrm{kg})$ and Morphine $4 \mathrm{mg}(0.08 \mathrm{mg} / \mathrm{kg})$ were given intravenously 15 minutes before the end of surgery. Total operation time for both cesarean section and abdominal aneurysm repair was 6 hours with an estimated blood loss of 4,000 mL. Extubation was delayed and the patient was transferred to the postanesthesia care unit (PACU) for monitoring and observation. Postoperative vitals signs were as follows: blood pressure of $130 / 80 \mathrm{mmHg}$, heart rate in the 120 s per minute, oxygen saturation at $99 \%$ and central venous pressure of 4 .

At the PACU, the patient's volume status was managed with additional blood and fluid replacement. She was extubated after four days at the intensive maternal unit and was eventually transferred to the surgical intensive care unit for further management. The patient was eventually discharged after three weeks while her baby boy stayed in the neonatal intensive care unit for further care.

\section{DISCUSSION}

Women are less likely to develop aortic aneurysm than men but they have a higher risk of rupture and mortality. The clinical diagnosis of abdominal aortic aneurysms is unreliable; hence, abdominal ultrasound is advised as safe, non-invasive, and readily available diagnostic tool with a sensitivity and specificity approaching 100\%. ${ }^{5}$ At present, there is no published local data on the incidence of abdominal aneurysm during pregnancy.

Available studies have conflicting results whether pregnancy causes an increase in aortic root diameter or not. Nonetheless, hemodynamic and hormonal changes associated with pregnancy place the patient at higher risk for suffering an aortic dissection. ${ }^{1}$ The proposed mechanism by which pregnancy lead to aortic complications involves hemodynamic changes that increase flow and turbulence within a closed vascular system (i.e. increases in heart rate, stroke volume, cardiac output, and left ventricular dimensions). This increase in flow proportionally increases shear stress exerted on the aortic wall. Shear stress is the tangential force exerted against the endothelium. High shear stress triggers vasodilation as a protective response against endothelial damage. In this sense, pregnancy actually decreases shear stress from a decrease in systemic vascular resistance and peripheral vasodilation. This is mediated by endothelium-dependent factors like nitric oxide that is upregulated by estradiol and prostaglandins. However, estrogen receptors present in the aortic tissue suppress synthesis of collagen and elastin responsible for endothelial integrity thereby weakening the elastic fibers in the aortic wall. The condition where turbulent abdominal aortic flow is exacerbated is due to an increase in outflow resistance in the distal aorta from the compression of a gravid uterus. This explains why aortic aneurysm complications usually happen during the third trimester or early post-partum. The goal, therefore, is to maximize the development of the fetus while managing the parturient's hemodynamic changes from the enlarging uterus to prevent dissection or rupture.

The choice of pharmacologic agent is important because of teratogenicity. Coordination among the internist, obstetrician and pediatrician is important since the care for the fetus starts even before delivery. The optimal period for surgery is between the 13th and 28th week of pregnancy. When the gestational age reaches 28 weeks, cesarean section should be considered as with this case. The decision to do emergency cesarean section was because of the enlarging aneurysm $(77 \mathrm{~mm})$ with associated abdominal pain. The reason for urgent cesarean delivery for a pregnant woman with aortic dissection is to avoid further hemodynamic stress that results to progressive aortic expansion, and aortic rupture. ${ }^{6}$ Emergency cesarean delivery is generally recommended if the aortic diameter is $>45 \mathrm{~mm} .{ }^{1}$

In planning the anesthesia care, several factors must be considered perioperatively. It is an interplay of maternal and fetal consideration, surgical stress, anesthetic technique, and postoperative recovery.

Maternal considerations include identification and management of co-morbidities to minimize risks. Careful preoperative evaluation of the patient and good communication among the internist, anesthesiologist, obstetrician and vascular surgeon is needed to optimize the success of the operation. Interval monitoring of the identified aneurysm should be done because of the hemodynamic changes in different stages of pregnancy. Preoperative assessment should include electrolytes, pain threshold and airway assessment. Control of perioperative pain and blood pressure and anticipation of blood loss are essential in management. Aggressive preoperative fluid resuscitation is not recommended as it will only increase bleeding and dilute clotting factors, which is already the case in pregnancy. Parturient patients are predisposed to airway edema and fragile mucosa that should be considered in preparation for difficult intubation. 
Fetal considerations should include viability based on age of gestation. It is important to consider the longest possible stay in-utero to maximize fetal development.

The management of aortic aneurysm is complicated by pregnancy because some drugs used to control the blood pressure have teratogenicity. The timing and dose are important, and the risk-benefit ratio of these drugs should be weighed. During gestation, heart defects (atrial and ventricular septal defects) are associated with the early use in pregnancy of antihypertensives like angiotensin receptor blockers and angiotensin converting enzyme inhibitors while beta blockers increase the odds of having cardiovascular anomalies, cleft lip and palate anomalies, and neonatal defects during the first trimester of pregnancy. ${ }^{78}$ During surgery, anesthetic technique and agents used can also affect the outcome of delivered newborn where hypoxia and acidosis can have life-long results like cerebral palsy. In a small retrospective study on fetal outcomes, general anesthesia did not show superiority over regional anesthesia. ${ }^{1}$ Although cesarean section can be done by pure epidural anesthesia technique, because of the high risk of aneurysm rupture, planned rather than emergency intubation is ideal to address the maternal airway challenges stated earlier. In this case, epidural anesthesia was combined with general anesthesia to minimize the exposure of fetus to the depressing effects of intravenous and inhalational anesthetics. During general anesthesia, induction to delivery interval of more than 8 minutes and uterine incision to delivery interval of more than 3 minutes predispose to neonatal acidosis and greater incidence of low 1-minute Apgar score. ${ }^{9}$ In this case, the time from induction to delivery was 3 minutes and the resulting Apgar score is 7 on the first minute and 8 on the fifth minute. After the delivery of the fetus, the anesthesia technique was shifted several times throughout surgery in response to hemodynamic needs of the mother.

The surgical procedure itself may dictate anesthesia care for the patient. Vaginal delivery is restricted only to low-risk patients with an aortic diameter of less than $40 \mathrm{~cm}$. Surgical treatment during pregnancy is associated with high fetal mortality and late neurological impairment in 3-6\% of the children. ${ }^{1}$ It should only be considered if medical treatment cannot control progression of aortic dilation and/or the life of the mother is in danger. Emergency repair of abdominal aortic aneurysm is a high-risk procedure. Central line insertion and the use of other invasive monitoring techniques must be considered for this case. ${ }^{4}$ Surgical stress can affect the overall hemodynamic status at any point; hence, close intraoperative monitoring was facilitated with the insertion of an arterial line and a central venous pressure monitor in this patient.

The repair of abdominal aortic aneurysm involves major hemodynamic shifts from the blood loss and the procedure itself. Notable is the cross clamping of the aorta. The physiologic effects of this technique during surgery vary with the level of the clamp in relation to the main aortic branches.
Perfusion to the lower extremities and the visceral organs is dependent on collateral circulation while the clamp is applied. The application of the clamp increases the afterload of the heart causing an increase in arterial pressure, which can be attenuated by deepening the anesthesia and giving opioids, and with use of vasodilators, like nitroglycerin and sodium nitroprusside. ${ }^{6}$ These agents decrease the systolic blood pressure and allow fluid loading in preparation for clamp release, where an opposite decrease in blood pressure is expected from redistribution. The expected increase in blood pressure during cross-clamping did not occur in this case. Instead, the patient had a significant decrease in blood pressure necessitating inotropic support. This may be due to hypovolemia secondary to blood loss. Continuous infusion of blood and crystalloids was done to manage the hypovolemia. When the cross-clamp is released, there is an expected $70-80 \%$ decrease in vascular resistance leading to hypotension. Hypotension may also be caused by the pooling of blood in the lower extremities, ischemia-reperfusion injury, and the washout of anaerobic metabolites causing lactic acidosis. ${ }^{6}$ This may be attenuated by the gradual release of the clamps and by adequate volume loading prior to release of clamp. The use of vasoconstrictors and inotropes may also be necessary. Myocardial load increases during this phase leading to higher oxygen demand. Ensuring the balance between myocardial oxygen supply and demand prevents myocardial ischemia from happening. Hypothermia should be avoided as it is also associated with myocardial ischemia and dysrhythmias and contributes to coagulopathy, further blood loss, and increase in wound infections. Forced-air warming device was used to prevent hypothermia of this patient. ${ }^{4}$

Postoperative management involves close monitoring and intensive care. The decision to delay extubation is on a case-by-case basis. Postoperative goals include hemodynamic stability, adequate replacement of blood volume, correction of electrolyte imbalances and acid-base status and adequate pain control. Epidural anesthesia provides excellent pain control facilitating early extubation to decrease pulmonary complications; however, correction of bleeding parameters in patients with massive blood loss and fluid shifts should be considered to avoid complications. Early enteral nutrition and mobility are encouraged.

\section{CONCLUSION}

Perioperative anesthetic management is individualized. A parturient for major general surgery, such as the repair of an abdominal aortic aneurysm, poses challenges that complicates the physiologic changes of pregnancy. The anesthesia technique, either purely regional or general anesthesia, combined regional-general anesthesia using inhalational or intravenous agents, should be tailored to best fit the mother and the unborn baby. Careful consideration of the risks and benefits of the type of anesthesia to be used 
should be done. Lastly, although the physiologic responses correlate with the steps in surgery, peculiar responses may occur. The anesthesiologist must anticipate the effects of surgery and anesthesia and respond to perturbations with flexibility to shift from one technique or agent to another. Postoperatively, early recovery and discharge planning should be considered. Although there is increasing success in aortic abdominal surgery due to advancement of technology and efficiency of technique, the goal of choosing and combining anesthesia techniques remains the same: to ensure safety and optimize outcomes for all patients.

\section{Statement of Authorship}

RJOI participated in writing, review and editing original draft and MTBA revised the manuscript and approved the final version submitted.

\section{Author Disclosure}

Both authors declared no conflicts of interest in preparing this article.

\section{Funding Source}

No specific grant from any funding agency in the public, commercial or not-for-profit sectors was received in writing this paper.

\section{REFERENCES}

1. Mosquera LM, De Backer J. Managing aortic aneurysms and dissections during pregnancy. Expert Review of Cardiovascular Therapy. 2015 May;1(12):1-12.

2. Yuan SM. Aortic Dissection During Pregnancy: A Difficult Clinical Scenario. Clin Cardiol. 2013 Oct; 36(10): 576-84.

3. Hooman K, Roman MJ, Pitcher A, Devereux RB. Pregnancy and the Risk of Aortic Dissection or Rupture: A Cohort-Crossover Analysis. [Internet]. New York (NY) 2016 [cited 2017 June 18]. Available from: https://www.ncbi.nlm.nih.gov/pmc/articles/PMC4987245/ pdf/nihms801889.pdf.

4. Crowlishaw P, Telford R, Kumar N. Anesthesia for abdominal aortic surgery. [Internet]. 2007 [cited 2017 June 18]. Available from: https://www.aagbi.org/sites/default/files/61-Anaesthesia-for-aorticsurgery.pdf.

5. Mecalfe D, Holt PE, Thompson MM. Management of abdominal aortic aneurysm. BMJ 2011; 342 doi: https://doi.org/10.1136/ bmj.d1384

6. Mhamad Al-Hashimi JT. Anaesthesia for elective open abdominal aortic aneurysm repair. Continuing Education in Anesthesia Critical Care \& Pain. 2013 Dec 1;23(6):208-12.

7. van Gelder MM, Jong-van den Berg L, Roeleveld N. Drugs associated with teratogenic mechanisms. Part II: a literature review of the evidence on human risks. Human Reproduction. 2014 Jan 1;29(1):168-83.

8. Yakoob M, Bateman BT, Ho E, Hernandez-Diaz S, Franklin JM, Goodman JE, Hoban RA. The risk of congenital malformations associated with exposure to beta-blockers early in pregnancy: A meta-analysis. Human Reproduction. 2013 Oct 9;29(1):168-83.

9. Sanjay D, Ostheimer GW, Weiss JB, Brown WU, Alper MH. Neonatal Effect of Prolonged Anesthetic Induction for Cesarean Section. Obstetric and Hynecology [Internet]. 1981 Sept. 